\title{
SHh activity and localization is regulated by perlecan
}

\author{
Verónica Palma ${ }^{1}$, Héctor Carrasco ${ }^{2}$, Gisela Reinchisi ${ }^{1}$, Gonzalo Olivares ${ }^{2}$, Fernando Faunes ${ }^{2}$ and Juan Larraín ${ }^{2 *}$ \\ ${ }^{1}$ Center for Genomics of the Cell, Facultad de Ciencias, Universidad de Chile, Casilla 653, Santiago, Chile. \\ ${ }^{2}$ Millenium Nucleus in Regenerative Biology, Center for Aging and Regeneration, Faculty of Biological Sciences, P. Universidad Católica de Chile, Alameda 340, \\ Santiago, Chile.
}

\begin{abstract}
Proliferation and cell fate determination in the developing embryo are extrinsically regulated by multiple interactions among diverse secreted factors, such as Sonic Hedgehog (SHh), which act in a concentration-dependent manner. The fact that SHh is secreted as a lipidmodified protein suggests the existence of a mechanism to regulate its movement across embryonic fields. We have previously shown that heparan sulfate proteoglycans (HSPGs) are required for SHh binding and signalling. However, it was not determined which specific HSPG was responsible for these functions. Here we evaluated the contribution of perlecan on SHh localization and activity. To understand the mechanism of action of perlecan at the cellular level, we studied the role of perlecan-SHh interaction in SHh activity using both cell culture and biochemical assays. Our findings show that perlecan is a crucial anchor and modulator of SHh activity acting as an extracellular positive regulator of $\mathrm{SHh}$.
\end{abstract}

Running title: Perlecan modulates SHh activity. Key words: SHh/Morphogen gradients / Perlecan.

\section{INTRODUCTION}

Differentiating cells undergo developmentally regulated changes in cell-cell and cell-matrix adhesion that control proliferation, differentiation and migration through microenvironments. The diversity of HSPGs expression patterns, coupled with their interactions with the extracellular matrix (ECM), cell adhesion molecules and growth factors during development, has emphasized their critical importance in the regulation of these events. Classical biochemical and cell culture assays have implicated HSPGs as modulators of growth factor activity; they bind through its HS chains to a variety of growth factors and regulate their ability to signal (Carey, 1997; Larraín et al., 1998; Bernfield et al., 1999). Perlecan is one of the most ubiquitous and multifunctional ECM proteins. Genetic analyses have uncovered a role for perlecan in mediating hedgehog ( $\mathrm{Hh}$ ) and fibroblast growth factor (FGF) signaling both in vertebrates and invertebrates (Park et al., 2003; Giros et al., 2007).

Sonic hedgehog (SHh) belongs to the hedgehog ( $\mathrm{Hh}$ ) family proteins. These are secreted proteins that act as morphogens, controlling embryonic development and tissue homeostasis (Ingham and McMahon, 2001, Riobo and Manning, 2007, Komada et al., 2008). In addition to the role of SHH during development, $\mathrm{SHH}$ activity needs to be tightly regulated during adulthood, since its aberrant activation is reported to predispose to malignant types of tumors in different organs (Clement et al., 2007, Stecca and Ruiz i Altaba, 2009).

Hh proteins are synthesized as a $45-\mathrm{kDa}$ precursors, that are auto-catalytically processed in N-terminal (Hh-N) and C-terminal (Hh-C) fragments (Lee et al., 1994). The Hh-N fragment suffers two lipidic modifications that restrict its free diffusion and facilitate transport and signaling (Mann and Beachy, 2004). First, and together with auto-processing, a cholesterol moiety is covalently attached to the C-terminus of the Hh-N fragment (Porter et al., 1996) and then a palmitate groups is added to its N-terminus (Pepinsky et al., 1998) producing a fully processed and active $\mathrm{Hh}$ molecule (Hh-Np). Cellular responses to $\mathrm{Hh}$ are mediated by the Hh-binding receptor patched1 (Ptc1) and the cell-surface transducer smoothened (Smo). In the absence of $\mathrm{Hh}$, Smo is inactive, probably because it localizes to internal membranes. After Hh binding to Ptc, Smo is relocated to the cell surface and becomes active (Ingham and McMahon, 2001; Zhu et al., 2003). Once activated, Smo induces a complex series of intracellular reactions that target the Gli family of transcription factors and culminates in the activation of Hh-specific targets such as Ptc1, Gli1, n-myc among others (Ingham and McMahon, 2001, for review see Ruiz i Altaba et al., 2003).

Hh tissue spreading is controlled not only by lipid modifications but also by cell surface Hh-binding molecules and proteoglycans in the extracellular space. Even though the role of HSPG in the Hh pathway have been interpreted as an effect on diffusion recent results clearly indicate that they are required for proper $\mathrm{Hh}$ signalling in embryonic epidermis (Desbordes and Sanson, 2003) and in cell culture assays (Lum et al., 2003). Analysis of Perlecan loss of function in mice shed new light on its role during development. Perlecan knockout mice show hypoplastic tissue and abnormal patterning in the embryonic brain. Perlecan is expressed in the basal lamina of the developing neuroepithelium and most perlecan null embryos (Costell et al., 1999) exhibit exencephaly. Interestingly, perlecan seems to be required to concentrate SHh signalling in the developing brain (Giros et al., 2007).

How does perlecan affect SHh function? Does it simply sequester it or does it act through an interaction with the receptor complex? To further understand the mechanism by which perlecan regulates SHh activity at the cellular level, we decided to evaluate the functional importance of this interaction using cell culture and biochemical assays. In order to elucidate perlecan-SHh interaction on a large scale, a multipotent mesodermal cell line $(\mathrm{C} 3 \mathrm{H} 10 \mathrm{~T} 1 / 2$ clone 8 ,

\footnotetext{
* Corresponding author: Juan Larraín Millenium Nucleus in Regenerative Biology, Center for Aging and Regeneration, Faculty of Biological Sciences, P. Universidad Católica de Chile, Alameda 340, Santiago, Chile (Fax: 562-686-2824, E-mail: jlarrain@bio.puc.cl)
} 
hereafter referred to as 10T1/2; Reznikoff et al., 1973) was employed as a model system. This line has been widely studied in regard to its responsiveness to Hh proteins (Kinto et al., 1997; Nakamura et al., 1997; Pathi et al., 2001). Importantly, perlecan has been shown to strongly induce chondrogenic differentiation in these cells (French et al., 1999). To directly address the role of perlecan we also used perlecan null embryonic fibroblasts (Costell et al., 1999). This study was further complemented by analyzing the effects of perlecan-SHh interactions in a developmental context evaluating perlecan interaction with $\mathrm{SHh}$ in rat brain extracts.

\section{RESULTS AND DISCUSION}

Hh proteins are a family of secreted proteins, including the mammal homologue Sonic hedgehog (SHH), which regulates multiple developmental processes in specific tissues (Ingham and McMahon, 2001, Riobo and Manning, 2007). Genetic analysis in Drosophila embryos has demonstrated that heparan sulphate proteoglycans (HSPG) are important players in the regulation of $\mathrm{Hh}$ diffusion and signaling. On the cell surface, lipidated SHh monomers are organized into oligomers that are recruited to preexisting HSPG-rich membrane domains to form large visible freely diffusible multimeric clusters (Dierker et al 2009).

We have previously shown that in trunk explants obtained from rat embryos $\mathrm{SHh}$ interacts preferentially with two HSPGs, that based on its molecular weight could correspond to glypican and perlecan (Carrasco et al., 2005). In the present study we directly evaluated the role of perlecan in $\mathrm{SHH}$ function.

First we analyzed the interaction between perlecan and SHh. 10T1/2 fibroblasts, which are responsive to SHh signaling, were lysed and incubated overnight either with a control or SHh-Fc resin and the fraction bound to the resin afterwards was analyzed by immunoblot with anti-perlecan antibody. We found that SHh interacts with perlecan in mouse fibroblast extract since a band that matches the expected size was pulled down and recognized by the anti-perlecan antibody (Figure 1A). To demonstrate the interaction of $\mathrm{SHh}$ with perlecan under more physiological conditions, brain extracts from E13.5 rat embryos were incubated with anti-SHh specific antibodies (5E1) and the complexes formed were precipitated with protein A agarose beads. The immunoprecipitates were incubated with heparitinase and then separated in SDS-PAGE and developed with antibodies against perlecan (upper panel) and SHh (lower panel). Importantly, perlecan and SHh were co-immunoprecipitated (Figure 1B), indicating that perlecan and $\mathrm{SHh}$ interact in vivo during brain development. These bands were only precipitated when the anti-SHH antibody was present indicating that the interaction was specific. From these results we conclude that $\mathrm{SHh}$ and perlecan interact biochemically.

Since we previously showed that abrogating all HSPG action by using chlorate treatment resulted in a decrease in SHh binding and signaling (see Carrasco et al., 2005) we decided to establish the particular role of perlecan in SHh localization and activity. To test this we used perlecan null mouse embryonic fibroblasts (Costell et al., 1999). We verified the absence of perlecan in these cells by PCR, immunofluorescence (data not shown) and immunoblots with anti-perlecan and anti-stub specific antibodies (Figure
$2 \mathrm{~A})$. These analyses confirmed that the absence of perlecan is specific.

We reasoned that the ability of perlecan to bind SHh might regulate its cellular localization. To test this, we made use of the monoclonal anti-SHh 5E1 antibody that is commonly used to detect the biologically active morphogen. SHh was expressed in wild-type cells independently of previous incubation with TX-100 $0.1 \%$ (Figure 2B). Strikingly, no $\mathrm{SHh}$ protein was detected in perlecan null cells treated with detergent but its expression was normal in non-treated cells. This effect on $\mathrm{SHh}$ was not due defective expression of $\mathrm{SHh}$ (data not shown) or a general defect on cell-surface proteins, as Ptc1 localization was not affected in perlecan null cell (Figure 2B). Following this, we measured the binding of a SHh-alkaline phosphatase fusion protein (SHH-AP) to wild type and perlecan null cells. We found that in the absence of perlecan SHh-AP binds less efficiently to the cell surface (Figure 2C). In summary, we have found that perlecan is required to retain SHh associated with the cell.

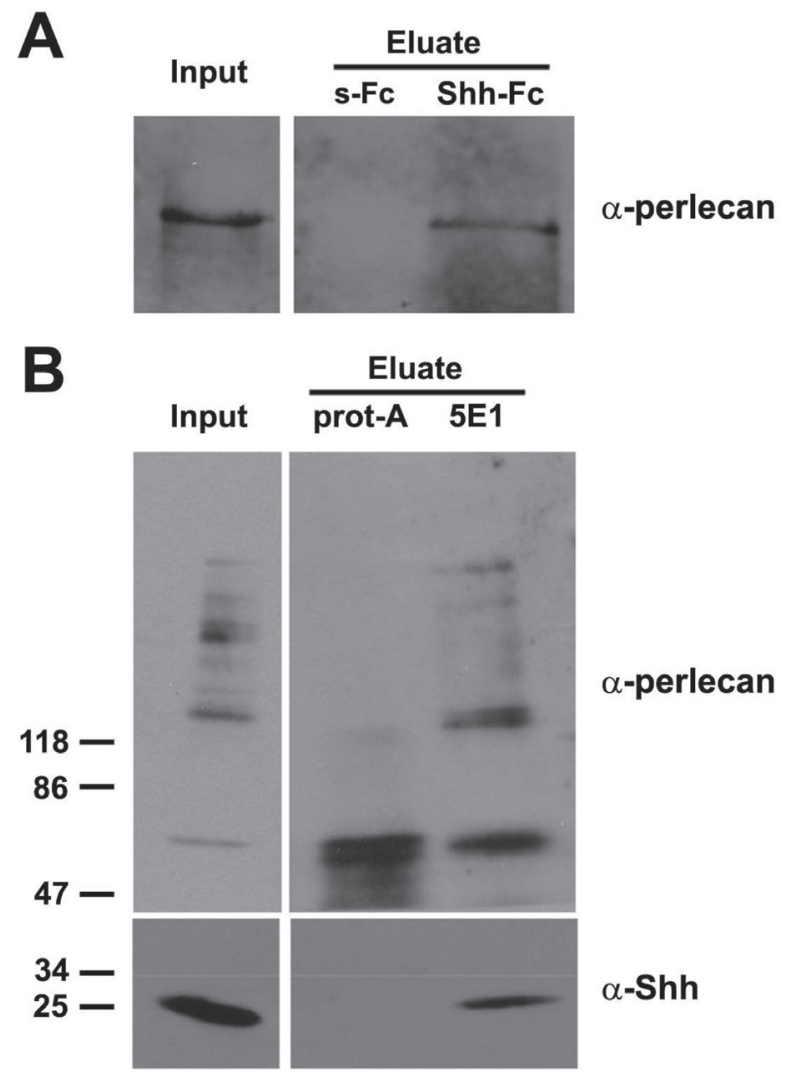

Figure 1. Perlecan interacts with SHH: A) 10T1/2 cells were lysed and incubated overnight with $\mathrm{SHh}-\mathrm{Fc}$ (protein-A coupled) and control resins (only protein- $A$ ) at $4^{\circ} \mathrm{C}$. The fraction bound to the resin was treated with heparitinase and analyzed by immunoblot with anti-perlecan antibody. B) Anti-SHh antibody (5E1) was crosslinked to protein-A resin and this column was incubated with E13.5 rat brain extracts. Column eluates were treated with heparitinase and loaded for immunoblot with anti-perlecan (upper gel) and anti-SHh antibody (lower gel). Bands between 170-300 $\mathrm{kDa}$ in $5 \mathrm{E} 1$ eluates correspond to perlecan, indicating that it coimmunoprecipites with SHh. 
To study the role of perlecan in SHh activity, we measured AP induction and made use of a well-described SHh specific luciferase reporter (pGli-luc) assay. We found that $\mathrm{SHh}$ was not able to induce any of these targets in perlecan null cells (Figure 3A,B). Nevertheless perlecan ${ }^{-/}$cells can still respond to Hh signaling since after co-transfection with a Gli2 expression construct (the main downstream activator in mice) we were able to observe pGli-luc induction, although this response was diminished (Figure 3C).

Our observations suggest perlecan is a strong regulator of SHh function, particularly in the brain (Figure 1B). Recent findings indicate that SHh-proteoglycan interactions mediated by the Cardin-Weinstraub motif are specifically required for growth regulation, but not for patterning activities in the embryonic brain (Chan et al., 2009). We have previously documented that SHh pathway activities increase presumptive stem cell and/or progenitor cells number by stimulating their proliferation and/or maintenance both in the developing dorsal brain, as well as in neurogenic niches in the adult (Palma and Ruiz i Altaba, 2004; Palma et al., 2005). Of note, the perlecan null dorsal brain phenotype resembles the one described for SHh ko and gli2 ko (Giros et al., 2007; Palma and Ruiz i Altaba, 2004). Thus, our analysis on the role of perlecan in SHh activity presented here suggests strongly that this HSPG is required for both localization and active signaling. We speculate that extracellular matrix composition of biological stem cell "niches" may play an important role in controlling context dependent SHh function in the CNS.
A

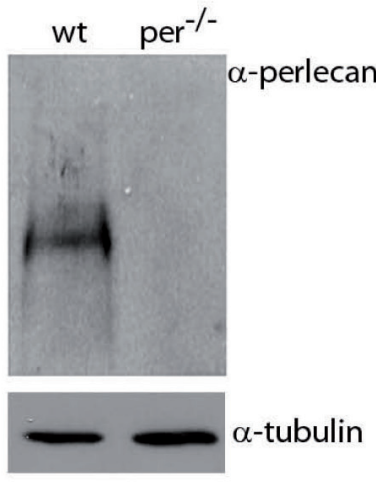

B

Shh
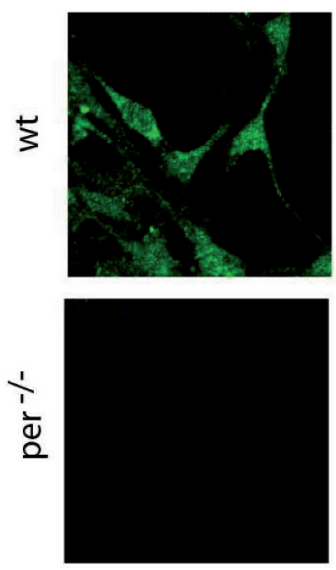

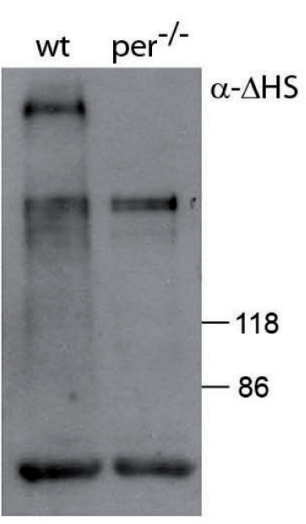

(+) TX-100

Ptc1
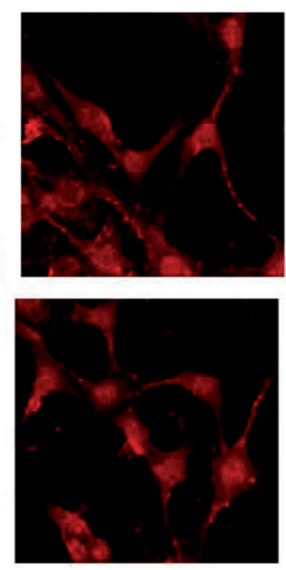

C

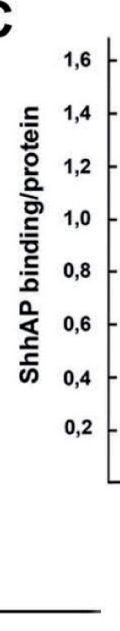

DAPI
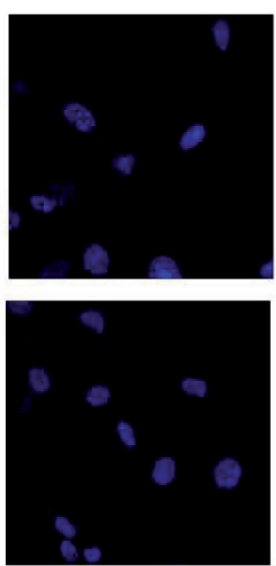

Shh
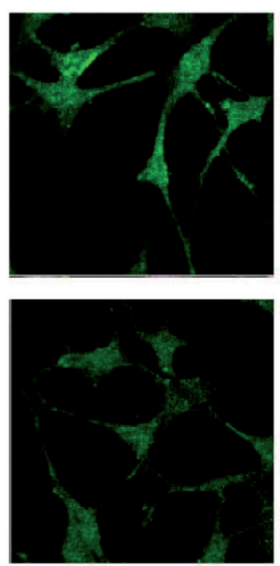
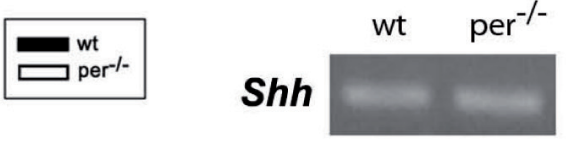

Hprt

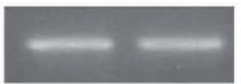

Figure 2. Cell associated SHh is altered in perlecan null cells: A) Immunoblot anti-perlecan (left gel) and anti-stub (right gel) of extracts from wt and perlecan $\%$ cells showed that perlecan null cells have no perlecan protein and other HSPG remain at normal levels.B) Wt and perlecan null cells were seeded and processed for immunofluorescence with anti-SHh and anti-Ptc 1 antibodies post permeabilization $(+\mathrm{TX}-100)$ or without permeabilization (-TX-100). Reaction for anti-SHh antibody is lost in perlecan $\%$ cells after permeabilization, but is not affected in perlecan $/-$ cells compared to wt cells without detergent. Ptc1 expression is maintained in perlecan $/-$ fibroblasts. C) SHHAP binding is reduced in perlecan $\%$ cells. Wt and perlecan $\%$ cells were grown for 3 days in 96-well plates, washed and incubated with different dilutions of SHHAP fusion protein for 2 hours at $4^{\circ} \mathrm{C}$. Then cells were lysed and bound AP activity and protein concentration determined. Figure shows that perlecan $\%$ cells bound less SHHAP than wt cells, indicating that perlecan is necessary for SHh binding to cell surface. Wt, wild type; per $^{--}$, perlecan null. Scale Bar $=10 \mathrm{mM}$. 
A

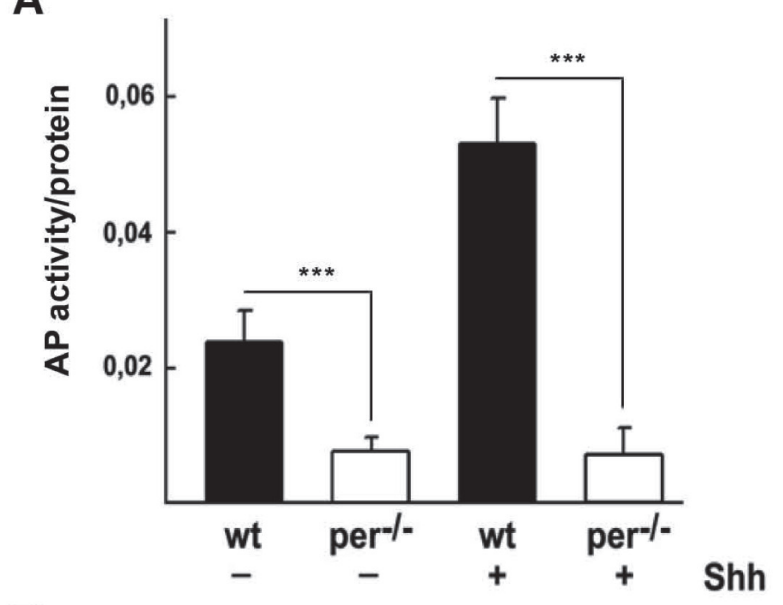

B

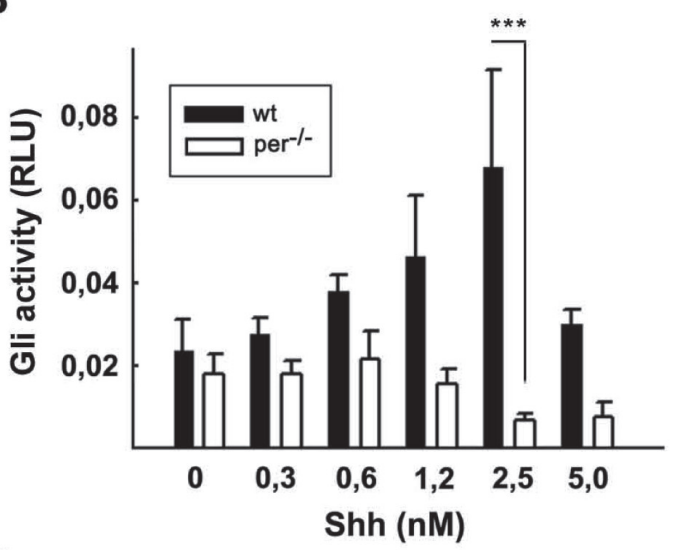

C

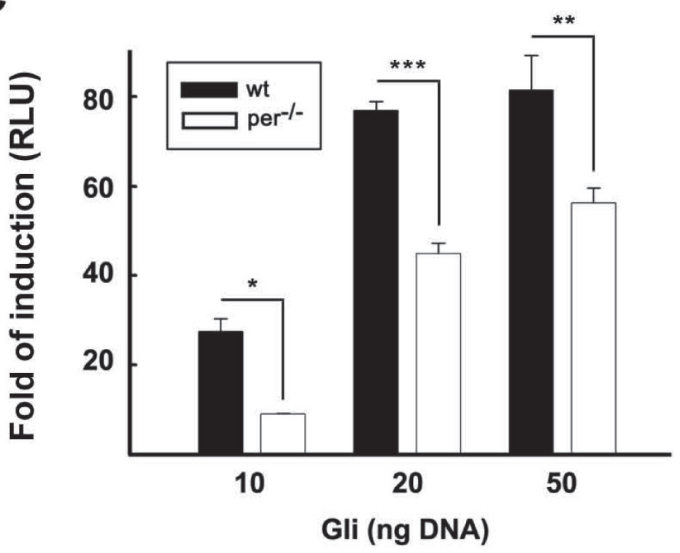

Figure 3. SHH response is disminished in perlecan-/- cells. A) Confluent wt and perlecan ${ }^{-1}$ cells were incubated with $\mathrm{SHH} 5$ $\mathrm{nM}$ in 96-well plates for 3 days and lysed. Alkaline phosphatase (AP) activity and protein concentration were determined in cell extracts and ratio plotted. Perlecan ${ }^{\%}$ cells have no SHh-induced AP activity respect to wt cells, indicating that this proteoglycan is necessary for SHh signa ling. B) Wt and perlecan ${ }^{\%}$ cells were transfected with SHH luciferase reporter (pGLi-luc) and control plasmid (pRL) and incubated with different concentration of $\mathrm{SHH}$ for 1 day. Cells were lysed and luminiscence determined. SHH induced pGli-luc in wt cells, but not in perlecan $\%$ cells. C) Wt and perlecan $\%$ cells were co-transfected with the SHH reporter PGLiluc and DNA for Gli2 and luminiscence was determined. Results indicate that perlecan $\%$ is still able to respond to Gli. Wt, wild type; per $^{-/}$, perlecan null.

\section{Concluding remarks}

The complexity of Hh signaling is daunting and key mechanistic questions are currently those of Hh presentation and transduction. Our findings show that SHh interacts with perlecan in both cell and rat brain extracts. Perlecan is not only necessary for proper localization of SHh in fibroblasts since our experiments with perlecan ko cells further demonstrate that perlecan is required for maximal SHh signaling.

Taken together, the results of our expression, inhibition, and biochemical studies link perlecan expression and function to SHH-Gli pathway activity in both SHh responding cell lines and in the developing brain.

\section{MATERIAL AND METHODS \\ Preparation of $P G$ enriched rat brain extracts}

Brains from rat E13.5 embryos were homogenized in buffer A (10 mM Tris pH 7.5, $150 \mathrm{mM} \mathrm{NaCl,} \mathrm{0.5 \%} \mathrm{TX-100}$ and protease inhibitors) and bound to anti-5E1 affinity columns. After binding overnight at $4^{\circ} \mathrm{C}$ the beads were washed extensively with the same buffer $\mathrm{A}$ and treated with heparitinase (overnight at $37^{\circ} \mathrm{C}$ ), after that beads were eluted and loaded in SDS-PAGE for western blot analysis. The antiSHh immunoaffinity column was prepared by coupling the 5E1 monoclonal antibody to AffiGel 10 (Pepinsky et al., 1998; Taipale et al., 2000).

\section{Protein purification and chromatography}

SHh-Np was purified by immunoaffinity chromatography from a human 293T cell line that express mouse SHh. SHHFc was collected from conditioned media of a 293T cell line that express SHh-Fc and s-Fc from a S2 Drosophila cell line and both were purified as described (Larraín et al., 2003). For preparation of affinity columns pure $\mathrm{SHH}-\mathrm{Fc}$ and s-Fc proteins were bound to protein-A agarose beads and crosslinked using dimethylpimelimidate (DMS) (Larraín et al., 2003). PGs enriched pools were incubated with SHH-Fc or s-Fc columns at $4^{\circ} \mathrm{C}$ for $16 \mathrm{~h}$ with rocking, then the beads were washed with buffer A containing $0.2 \mathrm{M}$ or $0.5 \mathrm{M} \mathrm{NaCl}$ until the radioactivity in the washout was cero and eluted with 3 column volumes of buffer A containing $1 \mathrm{M} \mathrm{NaCl}$. The eluates were dialyzed against buffer $\mathrm{A}$ and treated with $0.5 \mathrm{mU}$ of heparitinase (Seikagaku, Japan). The samples were separated by SDS-PAGE on a $4-15 \%$ gradient gel and analyzed by Western blot using an anti- $\Delta$-heparan sulfate antibody.

\section{Cell surface binding}

Wt or perlecan ${ }^{-/-}$fibroblasts were grown to $80 \%$ confluence in 96-well plates, washed twice with $0.2 \mathrm{ml}$ of ice-cold HBSS (Hank's Balanced Salt Solution containing BSA, $0.5 \mathrm{mg} / \mathrm{ml}$, and $20 \mathrm{mM}$ Hepes, $\mathrm{pH} 7.0$ ) and incubated for $2 \mathrm{~h}$ on ice with $50 \mu \mathrm{l}$ alkaline phosphatase (AP) fusion proteins. Cells were then washed on ice 6 times with $0.2 \mathrm{ml}$ of ice-cold HBSS and extracted with $60 \mu \mathrm{l}$ of $10 \mathrm{mM}$ Tris- $\mathrm{HCl} \mathrm{pH} 7.5$ containing $1 \%$ Triton X-100. Endogenous AP activity was inactivated by incubation of the plate at $65^{\circ} \mathrm{C}$ for $1 \mathrm{~h}$. AP activity resulting from fusion proteins bound to the cells was detected by a colorimetric AP assay. After $30 \mathrm{~min}$. of incubation at $37^{\circ} \mathrm{C}$, 
the reaction was stopped with $200 \mu \mathrm{l}$ of $0.5 \mathrm{M}$ glycine- $\mathrm{NaOH}$. Absorbance was read at $405 \mathrm{~nm}$ in a plate reader.

Alkaline phosphatase induction

SHH activity was tested measuring alkaline phosphatase induction in wt and perlecan ${ }^{-/}$fibroblasts based on Pepinsky et al., 1998. Cell were plated in 96-well plates and grown to confluence, SHh-Np or SHHN was added to the growth medium and alkaline phosphatase was measured as described after three days (Nakamura et al., 1997). In the experiment where the effect of the peptide was tested, fresh peptide and SHh-Np were added every day.

\section{RT-PCR and Immunofluorescence}

Samples from the perlecan deficient and wild type controls were processed for both RT-PCR and immunofluorescence. RNA preparation and RT-PCR conditions and sequences for the Hprt, Gli1 and SHh primer pairs were as described (Palma and Ruiz i Altaba, 2004). Primary antibodies used were: antiperlecan domain II, anti-SHh 5E1 (1/50, Hybridoma Bank), anti- Ptc1 (1/100, Santa Cruz).

Statistics

All probability values were obtained using the Student's t-test.

\section{ACKNOLEDGEMENTS}

We thank Dr. P. Beachy for the stable SHH line, Dr. C.M. Fan for the SHH-AP and SHH-Fc constructs. We are particularly grateful to Dr. Reinhard Fassler for the perlecan null fibroblasts. The 5E1 hybridoma was obtained from the Developmental Studies Hybridoma Bank from Iowa University. Thanks to V.H. for critical reading and editing of the manuscript. G.R. received a MECESUP fellowship. This work was supported by ICM P06-039F (VP), Fondecyt grant 1070248 (VP), Fondecyt grant 1030481 (JL).

\section{REFERENCES}

BERNFIELD, M., GOTTE, M., PARK, P. W., REIZES, O., FITZGERALD, M. L., LINCECUM, J., and ZAKO, M. (1999) Functions of cell surface heparan sulfate proteoglycans. Annu. Rev. Biochem., 68, 729-777.

CAREY, D.J. (1997) Syndecans: multifunctional cell- surface co-receptors. Biochemical, J., 327, 1-16.

CARRASCO H, OLIVARES GH, FAUNES F, OLIVA C, LARRAÍN J. (2005) Heparan sulfate proteoglycans exert positive and negative effects in Shh activity. J Cell Biochem. 96, 831-8.

CHAN J. A., BALASUBRAMANIAN S., WITT R. M., NAZEMI K. J., CHOI Y., PAZYRA-MURPHY M. F., WALSH C. O., THOMPSON M. and SEGAL R. A. (2009) Nat Neurosci, 12,409-417.

CLEMENT V, SANCHEZ P, DE TRIBOLET N, RADOVANOVIC I, RUIZ I ALTABA A. (2007) HEDGEHOG-GLI1 signaling regulates human glioma growth, cancer stem cell self-renewal, and tumorigenicity. Curr Biol. 17, 165-72.

COSTELL, M., GUSTAFSSON, E., ASZODI, A., MORGELIN, M., BLOCH, W., HUNZIKER, E., ADDICKS, K., TIMPL, R., and FASSLER, R. (1999) Perlecan maintains the integrity of cartilage and some basement membranes. J. Cell Biol., 147, 1109-1122.

DESBORDES, S. C. and SANSON, B. (2003) The glypican Dally-like is required for Hedgehog signalling in the embryonic epidermis of Drosophila. Development 130, 6245-6255.

DIERKER T, DREIER R, MIGONE M, HAMER S, GROBE K. (2009) Heparan sulfate and transglutaminase activity are required for the formation of covalently cross-linked hedgehog oligomers. J Biol Chem. 284, 32562-71.
FRENCH MM, SMITH SE, AKANBI K, SANFORD T, HECHT J, FARACHCARSON MC, CARSON DD. (1999) Expression of the heparan sulfate proteoglycan, perlecan, during mouse embryogenesis and perlecan chondrogenic activity in vitro. J Cell Biol. 145, 1103-15.

GIRÓS A, MORANTE J, GIL-SANZ C, FAIRÉN A, COSTELL M. (2007) Perlecan controls neurogenesis in the developing telencephalon. BMC Dev Biol. 7, 29.

INGHAM, P. W. and MCMAHON, A. P. (2001) Hedgehog signaling in animal development: paradigms and principles. Genes Dev., 15 3059-3087.

KINTO N, IWAMOTO M, ENOMOTO-IWAMOTO M, NOII S, OHUCHI H, YOSHIOKA H, KATAOKA H, WADA Y, YUHAO G, TAKAHASHI HE, YOSHIKI S, YAMAGUCHI A. (1997) Fibroblasts expressing Sonic hedgehog induce osteoblast differentiation and ectopic bone formation. FEBS Lett. 404, 319-23.

KOMADA M., SAITSU H., KINBOSHI M., MIURA T., SHIOTA K AND ISHIBASHI M. (2008) Hedgehog signaling is involved in development of the neocortex. Development., 135, 2717-2727.

LARRAÍN, J., CAREY, D. J., and BRANDAN, E. (1998). Syndecan-1 expression inhibits myoblast differentiation through a basic fibroblast growth factor-dependent mechanism. J. Biol. Chem. 273, 32288-32296.

LARRAÍN, J., BROWN, C. and DE ROBERTIS, E. M. (2003) Integrin- $\alpha 3$ mediates binding of Chordin to the cell surface and promotes its endocytosis. EMBO Reports, 4, 813-18.Lee, J.J., Ekker, S.C., Von Kessler, D.P., Porter, J.A., Sun, B.I. and Beachy, P.A. (1994) Autoproteolysis in hedgehog protein biogenesis. Science, 266, 1528-1537.

LUM, L., YAO, S., MOZER, B., ROVESCALLI, A., VON KESSLER, D. NIRENBERG, M., and BEACHY, P. A. (2003) Identification of Hedgehog pathway components by RNAi in Drosophila cultured cells. Science, 299, 2039-2045.

MANN, R. K. and BEACHY, P. A. (2004) Novel lipid modifications of secreted protein signals. Ann. Rev. Biochem., 73, 891-923.

NAKAMURA, T., AIKAWA, T., IWAMOTO-ENOMOTO, M., IWAMOTO, M., HIGUCHI, Y., PACIFICI, M., KINTO, N., YAMAGUCHI, A., NOJI, S., KURISU, K., and MATSUYA, T. (1997) Induction of osteogenic differentiation by hedgehog proteins. Biochem. Biophys. Res. Commun. 237, 465-469.

PALMA V, RUIZ I ALTABA A. (2004) Hedgehog-GLI signaling regulates the behavior of cells with stem cell properties in the developing neocortex. Development. 131, 337-45.

PALMA V, LIM DA, DAHMANE N, SÁNCHEZ P, BRIONNE TC, HERZBERG CD, GITTON Y, CARLETON A, ALVAREZ-BUYLLA A, RUIZ I ALTABA A. (2005) Sonic hedgehog controls stem cell behavior in the postnatal and adult brain. Development. 132, 335-44.

PARK, Y., RANGEL, C., REYNOLDS, M. M., CALDWELL, M. C., JOHNS, M., NAYAK, M., WELSH, C. J., MCDERMOTT, S., and DATTA, S. (2003) Drosophila perlecan modulates FGF and hedgehog signals to activate neural stem cell division. Dev Biol., 253, 247-257.

PATHI S, PAGAN-WESTPHAL S, BAKER DP, GARBER EA, RAYHORN P, BUMCROT D, TABIN CJ, BLAKE PEPINSKY R, WILLIAMS KP. (2001) Comparative biological responses to human Sonic, Indian, and Desert hedgehog. Mech Dev. 106, 107-17.

PEPINSKY, R. B., ZENG, C., WEN, D., RAYHORN, P., BAKER, D. P., WILLIAMS, K. P., BIXLER, S. A., AMBROSE, C. M., GARBER, E. A., MIATKOWSKI, K., TAYLOR, F. R., WANG, E. A., GALDES, A. (1998) Identification of a palmitic acid-modified form of human Sonic hedgehog.J. Biol. Chem., 273, 14037-14045.

PORTER, J. A., YOUNG, K. E., and BEACHY, P. A. (1996) Cholesterol modification of hedgehog signaling proteins in animal development. Science, 274, 255-259.

REZNIKOFF CA, BRANKOW DW, HEIDELBERGER C. (1973) Establishment and characterization of a cloned line of $\mathrm{C} 3 \mathrm{H}$ mouse embryo cells sensitive to postconfluence inhibition of division. Cancer Res. 33, 3231-8.

RIOBO NA, MANNING DR. (2007) Pathways of signal transduction employed by vertebrate Hedgehogs. Biochem J. 403, 369-79.

RUIZ I ALTABA A, NGUYÊN V, PALMA V. (2003) The emergent design of the neural tube: prepattern, SHH morphogen and GLI code. Curr Opin Genet Dev. 13, 513-21.

STECCA B. AND RUIZ I ALTABA A. (2009) A GLI1-p53 inhibitory loop controls neural stem cell and tumour cell numbers. EMBO J. 28, 663-676.

TAIPALE, J., CHEN, J. K., COOPER, M. K., WANG, B., MANN, R. K., MILENKOVIC, L., SCOTT, M. P. and BEACHY, P. A. (2000) Effects of oncogenic mutations in Smoothened and Patched can be reversed by cyclopamine. Nature, 406, 1005-1009.

ZHU, A. J., ZHENG, L., SUYAMA, K., SCOTT, M. P. (2003) Altered localization of Drosophila Smoothened protein activates Hedgehog signal transduction. Genes Dev., 17, 1240-1252. 
